\title{
CLOSED PRIMARY IDEALS IN A CLASS OF BANACH ALGEBRAS ${ }^{1}$
}

\author{
YNGVE DOMAR
}

1. Preliminaries. Our main purpose is to prove two theorems (Theorem 1 and Theorem 2) on the primary ideal structure in a class of Banach algebras which was introduced and discussed by Beurling [1] and Wermer [6] and then studied in a larger context in [3]. As corollaries we obtain results on integral equations of convolution type (Theorem 4) and on polynomial approximation (Theorem 5). For the proof of Theorem 1 we need a property of entire functions of exponential type. Since it may be of independent interest, it is stated as a separate theorem (Theorem 3).

We shall start by defining a Banach algebra of the type under question and then we shall enlist, referring to [3], those of its properties which we need for the discussion.

Let $p$ be a Lebesgue measurable real-valued function on the real line $-\infty<x<\infty$. We assume that $p$ is bounded on every compact interval, that

$$
p(x) \geqq 1,
$$

for every $x$, that

$$
p\left(x_{1}+x_{2}\right) \leqq p\left(x_{1}\right) p\left(x_{2}\right),
$$

for every $x_{1}$ and $x_{2}$, and finally that

$$
\int_{-\infty}^{\infty} \frac{\log p(x)}{1+x^{2}} d x<\infty .
$$

We form the Banach space $F_{p}$ of complex-valued Lebesgue measurable functions $f$ with the finite norm

$$
\int_{-\infty}^{\infty}|f(x)| p(x) d x .
$$

Received April 8, 1959.

1 Lecture given at the conference on harmonic analysis at Gothenburgh November 29-30, 1958. 
The condition (2) guarantees that $F_{p}$ is a Banach algebra under the commutative convolution operation

$$
f * g=\int_{-\infty}^{\infty} f(x-y) g(y) d y .
$$

It is shown in [3, Ch. 1 and 2], that $F_{p}$ has the dual real line $-\infty<t<\infty$ as its space of regular maximal ideals. The Gelfand representation of an element $f \in F_{p}$ is given by its Fourier transform

$$
\hat{f}(t)=\int_{-\infty}^{\infty} e^{-i t x} f(x) d x .
$$

Thus to every real $t$ there corresponds a regular maximal ideal $I_{0}(t)$, consisting of the elements $f \in F_{p}$, for which $\hat{f}(t)=0$. Conversely, we obtain in this way every regular maximal ideal. $F_{p}$ is regular, i.e. to every real $t_{0}$ and every $\varepsilon>0$ there exists an element $f \in F_{p}$ such that $\hat{f}\left(t_{0}\right) \neq 0$, while $\hat{f}(t)=0$ if $\left|t-t_{0}\right|>\varepsilon$. Furthermore, $F_{p}$ has the following fundamental property (the generalized Tauberian theorem, cf. [3, Theorem 1.53]):

A. Every proper closed ideal is included in at least one regular maximal ideal.

The space $F_{p}^{*}$ of bounded linear functionals on $F_{p}$ can be identified with the class of measurable complex-valued functions $\varphi$ on $-\infty<x<\infty$ such that

$$
\sup _{x} \frac{|\varphi(x)|}{p(-x)}<\infty,
$$

in the sense that the value for $f \in F_{p}$ of the corresponding functional is

$$
\int_{-\infty}^{\infty} \varphi(-x) f(x) d x .
$$

The spectrum $\Lambda_{\varphi}$ for a function $\varphi \in F_{p}^{*}$ is defined as the set of all $t$ for which the corresponding ideal $I_{0}(t)$ contains every $f \in F_{p}$ for which

$$
\varphi * f \equiv 0 \text {. }
$$

(See [3, Ch. 3.1]). For any $\varphi \in F_{p}^{*}$ and any $f \in F_{p}$ we have

$$
\varphi * f \in F_{p}^{*},
$$

and if $f^{\prime}$ is another element in $F_{p}$, we have

$$
\varphi *\left(f * f^{\prime}\right) \equiv(\varphi * f) * f^{\prime} .
$$


This shows that for any given $\varphi \in F_{p}^{*}$ the class of all $f \in F_{p}$ which satisfy (4), is a closed ideal. Hence the property $A$ has the following consequence:

B. $\Lambda_{\varphi}$ is empty if and only if $\varphi \equiv 0$ almost everywhere. $([3$, Theorem $3.21 \mathrm{~A}]$.)

We need moreover the following results from [3]:

C. Suppose that $f \in F_{p}$ and $\varphi \in F_{p}^{*}$ and that $\hat{f}(t)$ vanishes on a set which contains $A_{\varphi}$ in its interior. Then

\section{([3, Theorem 3.21 D].)}

$$
\varphi * f \equiv 0 .
$$

D. Suppose that $\varphi \in F_{p}^{*}$ has $\Lambda_{\varphi}$ consisting only of the point $t=0$. Then $\varphi$ coincides almost everywhere with an entire function of exponential type 0 ([3, Theorem 3.35].). For any positive integer $n$ we denote the $n$-th derivative of this function by $\varphi^{(n)}$. Then

$$
\varphi^{(n)} \in F_{p}^{*},
$$

and if (4) holds for a function $f \in F_{p}$, this implies that

$$
\varphi^{(n)} * f \equiv 0 .
$$

([3, Theorems 3.31 and 3.34].)

2. Primary ideals. Statement of the main theorems. A primary ideal in $F_{p}$ is an ideal, which is included in exactly one regular maximal ideal. We shall below introduce certain additional assumptions on $p$, which have as a consequence that $p(x)$ grows to infinity faster than any polynomial, when $|x| \rightarrow \infty$. This implies that to every regular maximal ideal $I_{0}(t)$ there corresponds an infinite number of closed primary ideals. For to any positive integer $n$ we obtain such an ideal if we form the class $I_{n}(t)$ of all $f \in F_{p}$ such that

$$
\hat{f}(t)=\hat{f}^{\prime}(t)=\ldots=\hat{f}^{(n)}(t)=0 .
$$

The rapidity of growth of $p$ then guarantees both the existence of these derivatives and the closure property of the ideals. That they are primary is a consequence of the regularity of $F_{p}$. Another closed primary ideal, corresponding to $t$, is

$$
I_{\infty}(t)=\bigcap_{n} I_{n}(t)
$$

We can now state the main theorems: 
THEOREM 1. If $p$ fulfills the additional assumptions 1 below and if $I(t)$ is a closed primary ideal, included in $I_{0}(t)$ but not in $I_{\infty}(t)$, then it has to coincide with one of the ideals $I_{n}(t), n=0,1,2, \ldots$

THEOREM 2. If $p$ fulfills the additional assumptions 2 below and if $I(t)$ is a closed primary ideal, included in $I_{\infty}(t)$, then it has to coincide with $I_{\infty}(t)$.

We have the following immediate consequence of these theorems:

CoRollaRY. If $p$ fulfills the additional assumptions 1 and 2 below, then any closed primary ideal in $F_{p}$ is of the form $I_{n}(t)$, where $n$ is a non-negative integer or $\infty$.

We shall now give the additonal assumptions which we need. Especially the assumptions 1 look very restrictive, but it should be observed that the restrictions concern the smoothness more than the rapidity of growth. Both of the additional assumptions are for instance fulfilled if

or if $p$ coincides with

$$
p(x)=e^{|x|^{\alpha}}, \quad 0<\alpha<1,
$$

for large $|x|$.

$$
\exp \left\{\frac{|x|}{(\log |x|)^{\beta}}\right\}, \quad \beta>1,
$$

AdDitional ASSUMPTIONS 1. Let us suppose that there exist, for every positive integer $n$, positive numbers $x_{n}$ and $m_{n}$, such that

$1^{\circ}$ the $n$-th derivative $p^{(n)}(x)$ exists and

if $|x| \geqq x_{n}$,

$$
p^{(n)}(x) x^{n}>0,
$$

$2^{\circ}$

$$
\left|p^{(n)}(x)\right| \geqq 1,
$$

if $|x| \geqq x_{n}$,

$3^{\circ}$ the inequality

$$
\left|p^{(n)}\left(x^{\prime}+x^{\prime \prime}\right)\right| \leqq\left|p^{(n)}\left(x^{\prime}\right)\right|\left|p^{(n)}\left(x^{\prime \prime}\right)\right|
$$

holds if $\left|x^{\prime}\right| \geqq x_{n},\left|x^{\prime \prime}\right| \geqq x_{n}$ and $\left|x^{\prime}+x^{\prime \prime}\right| \geqq x_{n}$,

$4^{\circ}$ with $q(x)=\log p(x)$,

$$
a_{n}(x)=\left(\frac{p(x)}{\left|p^{(n)}(x)\right|}\right)^{1 / m_{n}} \text { and } \frac{1}{a_{n}(x)\left|q^{\prime}(x)\right|}
$$

are monotonically decreasing when $x \leqq-x_{n}$ and monotonically increasing when $x \geqq x_{n}$, 
$5^{\circ}$ the statement

$$
\int_{|x|>x_{n}}\left[q\left(x a_{n}(x)\right)+x q^{\prime}(x) a_{n}(x)\right] \frac{d x}{1+x^{2}}<\propto
$$

holds true. We may obviously assume that $x_{n}$ is monotonically increasing with $n$.

AdDitional ASSUMPTION 2. Let us suppose that $p$ is even and that $\log p(x)$ is a convex function of $\log |x|$ for large $|x|$, and finally that

$$
\frac{\frac{d}{d x} \log p(x)}{x^{-1} \log |x|} \rightarrow+\infty \quad \text { as } \quad|x| \rightarrow \infty .
$$

3. A theorem on entire functions of exponential type 0 . In order to prove Theorem 1 , we need the following theorem:

Theorem 3. Suppose that $s(x)>0$ is monotonically decreasing when $x \leqq 0$ and monotonically increasing when $x \geqq 0$. Suppose furthermore that the positive function $b(x)$ has the property that

$$
r(y)=\sup _{x}[\log s(x+y b(x))-\log s(x)]
$$

satisfies

$$
\int_{-\infty}^{\infty} \frac{r(y)}{1+y^{2}} d y<\infty
$$

Then there exists, for any positive integer $m$, a finite constant $\boldsymbol{M}_{\boldsymbol{m}}$ such that

$$
\sup _{x} \frac{\left|\varphi^{(m)}(x)\right|[b(x)]^{m}}{s(x)} \leqq M_{m} \sup _{x} \frac{|\varphi(x)|}{s(x)},
$$

for any entire function $\varphi$ of exponential type 0 and such that the right hand member is finite.

Proof of Theorem 3. The function $r(y)$ is apparently monotone for $y<0$ and for $y>0$. Using (6) and the arguments in Paley-Wiener $[5, \S 10]$, it is easy to see that we can find a not identically vanishing entire function $g$ of exponential type 1 such that

and

$$
\sup _{y}|g(y)| e^{r(y)}<\infty
$$

$$
\int_{-\infty}^{\infty}|g(y)| e^{r(y)} d y<\infty .
$$


It is obviously possible to choose $g$ in such a way that

and

$$
g(0)=1
$$

$$
g^{\prime}(0)=\ldots=g^{(m)}(0)=0 .
$$

Let $P(y)$ be a finite trigonometrical polynomial

$$
\sum a_{\nu} e^{i \lambda_{\nu} y}
$$

with every $\lambda_{\nu}$ real and $>1$, and such that at $y=0$

$$
P(y)=1+O\left(y^{m+1}\right) \text {. }
$$

Then we form the function

Apparently

$$
h(y)=g(y) \frac{\operatorname{Im}\{P(y)\}}{y^{m+1}} .
$$

$$
\int_{-\infty}^{\infty}|h(y)| e^{r(y)} d y=C<\infty .
$$

Further we introduce the expression

$$
\frac{m !}{\pi} \int_{-\infty}^{\infty} \varphi(x+y b(x)) h(y) d y,
$$

where $\varphi$ is any entire function of exponential type 0 such that

Then (9) can be written

$$
\sup _{x} \frac{|\varphi(x)|}{s(x)}=C_{0}<\infty .
$$

$$
\frac{m !}{\pi} \int_{\Gamma} \varphi(x+z b(x)) \frac{g(z)}{2 i z^{m+1}}(P(z)-\overline{P(\bar{z})}) d z,
$$

where $\Gamma$ denotes the real axis of the complex $z$-plane taken in the direction $-\infty$ to $\infty$. Formulas (5) and (7) imply that for every $x$ the function

$$
\varphi(x+z b(x)) g(z)
$$

has a bounded absolute value on the real axis. Since it is an entire function of exponential type 1, a well-known theorem (see for instance Boas [2, Theorem 6.2.4]) shows that

$$
|\varphi(x+z b(x))||g(z)| e^{-|\operatorname{Im}(z)|}
$$

is bounded in the complex plane. The integral (10) can be evaluated by changing $\Gamma$ in such a way that it avoids the point $z=0$, and then splitting 
the integral into the two parts which contain $P(z)$ and $\overline{P(\bar{z}})$, respectively. Owing to our assumption that every $\lambda_{v}>1$, these two integrals can be computed by means of simple residue calculus. We obtain

$$
\frac{m !}{\pi} \int_{-\infty}^{\infty} \varphi(x+y b(x)) h(y) d y=\varphi^{(m)}(x)[b(x)]^{m} .
$$

On the other hand the modulus of the left hand member of (11) is

$$
\leqq C_{0} \frac{m !}{\pi} \int_{-\infty}^{\infty} s(x+y b(x))|h(y)| d y,
$$

and by (5) and (8) this is in turn

$$
\leqq C_{0} \frac{m !}{\pi} s(x) \int_{-\infty}^{\infty} e^{r(y)}|h(y)| d y=C C_{0} \frac{m !}{\pi} s(x) .
$$

Hence

$$
\left|\varphi^{(m)}(x)\right|[b(x)]^{m} \leqq C C_{0} \frac{m !}{\pi} s(x),
$$

which proves the theorem.

REMARK. If the function $s$ in Theorem 3 moreover satisfies

for every $x$, and

$$
s(x) \geqq 1,
$$

$$
s\left(x_{1}+x_{2}\right) \leqq s\left(x_{1}\right) s\left(x_{2}\right),
$$

for every $x_{1}$ and $x_{2}$, then since

$$
\int_{-\infty}^{\infty} \frac{\log s(x)}{1+x^{2}}<\infty
$$

obviously is fulfilled, the classes $F_{s(-x)}$ and $F_{s(-x)}^{*}$ have the properties which were announced in Section 1 . Using property $D$ in Section 1 we see that the condition laid on $\varphi$ in Theorem 3 follows if we assume that

$$
\varphi \in F_{s(-x)}^{*}
$$

and that $\Lambda_{\varphi}$ consists at most of the point $t=0$. It should be observed that this version of the theorem can be proved by Fourier analysis arguments similar to those used in [3]. This makes it possible to extend the theorem to more general locally compact Abelian groups. 


\section{Consequences of the additional assumptions 1 .}

Lemma 1. For any positive integer $n$ let $c_{n}$ be a constant such that

$$
c_{n} \geqq 1, \quad c_{n} \geqq\left|p^{(n)}\left(-x_{n+1}\right)\right|, \quad c_{n} \geqq p^{(n)}\left(x_{n+1}\right) .
$$

We form the function $p_{n}$ defined by the relations

$$
p_{n}(x)=\left\{\begin{array}{lll}
c_{n}, & \text { if }|x| \leqq x_{n+1} \\
\max \left(c_{n}, p^{(n)}(x)\right), & \text { if }|x|>x_{n+1}
\end{array} .\right.
$$

Then the inequalities (1), (2) and (3) are fulfilled if $p$ is substituted by $p_{n}$. The function $p_{n}(x)$ is moreover monotonically decreasing if $x<0$ and monotonically increasing if $x>0$. Finally, if $m>n$,

$$
p_{m}(x)=O\left(p_{n}(x)\right), \quad \text { as } \quad|x| \rightarrow \infty,
$$

We omit the proof since the statements are immediate consequences of (1), (2), (3) and of $1^{\circ}, 2^{\circ}$ and $3^{\circ}$ in the additional assumptions 1 .

Lemma 2. Suppose that $f \in F_{p}$ and that

$$
\hat{f}(0)=\ldots=\hat{f}^{(n-1)}(0)=0 .
$$

Then

$$
f^{(-n)}(x)=\frac{1}{(n-1) !} \int_{-\infty}^{x}(x-y)^{n-1} f(y) d y
$$

belongs to the class $F_{p_{n}}$.

Proof. We shall only give a brief sketch of the proof. Using repeated integration by parts it is easy to show that

$$
\int_{-\infty}^{0}\left|f^{(-n)}(x)\right| p_{n}(x) d x<\infty .
$$

Formula (13) implies, however, that

Hence

$$
\int_{-\infty}^{\infty}(x-y)^{n-1} f(y) d y=0 .
$$

$$
f^{(-n)}(x)=-\frac{1}{(n-1) !} \int_{x}^{\infty}(x-y)^{n-1} f(y) d y,
$$

and using this formula the statement

$$
\int_{0}^{\infty}\left|f^{(-n)}(x)\right| p_{n}(x) d x<\infty,
$$

may be proved in a similar way. 
Lemma 3. If $\varphi \in F_{p}^{*}$, and if $\Lambda_{\varphi}$ consists only of the point $t=0$, then for any $n>0$ we have

$$
\varphi^{(m)} \in F_{p_{n}}^{*}
$$

if $m \geqq m_{n}$, where $m_{n}$ is defined in the additional assumptions 1 .

Proof. In the additional assumptions 1 we have defined the function $q(x)$ for every real $x$ and the functions $a_{n}(x)$ for every positive integer $n$ in case $|x| \geqq x_{n}$. We extend the definition of the functions $a_{n}(x)$ by defining them as an arbitrary positive constant $d$, if $|x| \leqq x_{n}$. We shall prove that

$$
r_{n}(y)=\sup _{x}\left[q\left(x+y a_{n}(x)\right)-q(x)\right]
$$

satisfies

$$
\int_{-\infty}^{\infty} \frac{r_{n}(y)}{1+y^{2}} d y<\infty .
$$

Let us first assume that $x \geqq 0$ and $y \geqq 0$. If $x \leqq x_{n}$ it follows from (2) that

$$
q\left(x+y a_{n}(x)\right)-q(x) \leqq q\left(y a_{n}(x)\right)=q(y d) .
$$

Since $q(x)$ is monotonically increasing if $x>x_{n},(2)$ and $4^{\circ}$ in the additional assumptions 1 give, if $x_{n}<x \leqq y$,

$$
q\left(x+y a_{n}(x)\right)-q(x) \leqq q\left(y a_{n}(x)\right) \leqq q\left(y a_{n}(y)\right) .
$$

By Lagrange's mean value therem and $4^{\circ}$ in the additional assumptions 1 we have furthermore, if $y<x$ and $x>x_{n}$,

where

$$
q\left(x+y a_{n}(x)\right)-q(x) \leqq y a_{n}(x) q^{\prime}(x) \leqq y a_{n}\left(y^{\prime}\right) q^{\prime}\left(y^{\prime}\right),
$$

$$
y^{\prime}=\max \left(y, x_{n}\right) \text {. }
$$

Quite similar results can be proved if one or both of $x$ and $y$ are negative. Hence by $(3)$ and $5^{\circ}$ in the additional assumptions 1 we see that (14) holds.

We now apply Theorem 3 with $p$ and $a_{n}$ as the functions $s$ and $b$, respectively. We then obtain (cf. the remark following the theorem) that

$$
\sup _{x} \frac{\left|\varphi^{(m)}(-x)\right|\left[a_{n}(x)\right]^{m}}{p(x)}<\infty
$$

for every $m$ and $n$, if $\varphi \in F_{p}^{*}$ and if $\Delta_{\varphi}$ consists of the only point $t=0$. Hence, if furthermore $m \geqq m_{n}$, we have by $4^{\circ}$ in the additional assumptions 1 , that

$$
\sup _{x} \frac{\left|\varphi^{(m)}(-x)\right|}{p_{n}(x)}<\infty,
$$

which proves the lemma. 
5. Proof of Theorem 1. Let us first observe that we can assume that $t=0$, for by means of mappings of the form

$$
f(x) \rightarrow f(x) e^{i t_{0} x}
$$

we see that we have isomorphic primary ideal structures at the various ideals $I_{0}(t)$.

Let us assume that $I(0)$ is a closed primary ideal, corresponding to $t=0$, but not included in $I_{\infty}(0)$. Closed ideals, being closed linear subspaces of the Banach space $F_{p}$, are completely characterized by the set of bounded linear functionals which vanish on them. For the ideals $I_{n}(0)$ these functionals are the derivatives of $\hat{f}(t)$ at $t=0$ up to the order $n$ and linear combinations of them. These functionals correspond to the class of functions $\varphi \in F_{p}^{*}$, which are equivalent to polynomials of degree $\leqq n$. Hence, if we can prove that the only functions $\varphi \in F_{p}^{*}$, which satisfy

$$
\int_{-\infty}^{\infty} \varphi(-x) f(x) d x=0
$$

for every $f \in I(0)$, are equivalent to polynomials with a uniformly bounded degree, then the theorem is proved. It is easy to prove by standard convolution arguments that every closed ideal in $F_{p}$ is invariant under translation. Hence the theorem is a consequence of the following proposition:

Proposition. The only functions $\varphi \in F_{p}^{*}$ which satisfy

$$
\varphi * f \equiv 0
$$

for every $f \in I(0)$, are those equivalent to polynomials of a certain uniformly bounded degree.

Proof of THE Proposition. Since $I(0)$ is a primary ideal, $\Lambda_{\varphi}$ consists only of the point $t=0$ or is empty. From $D$ in Section 1 we therefore conclude that for every positive integer $m$ and for every $f \in I(0)$

$$
\varphi^{(m)} * f \equiv 0 .
$$

Let $n$ be the smallest positive integer such that

This means that

$$
I(0) \notin I_{n}(0) \text {. }
$$

$$
\hat{f}(0)=\hat{f}^{\prime}(0)=\ldots=\hat{f}^{(n-1)}(0)=0
$$


for every $f \in I(0)$, whereas there exists at least one function $f_{0} \in I(0)$, such that

$$
\hat{f}_{0}^{(n)}(0) \neq 0 .
$$

By Lemma 3 all the functions

$$
\varphi^{\left(m_{n}\right)}, \varphi^{\left(m_{n}+1\right)}, \ldots, \varphi^{\left(m_{n}+n\right)}
$$

belong to the class $F_{p_{n}}^{*}$, and by (15), Lemma 2 and (12) all the functions

$$
f, f^{-1}, \ldots, f^{(-n)},
$$

belong to the class $\boldsymbol{F}_{p_{n}}$. Hence starting from the relation

$$
\varphi^{\left(m_{n}\right)} * f \equiv 0,
$$

which is valid for every $f \in I(0)$, and integrating $n$ times by parts, we obtain

$$
\varphi^{\left(m_{n}+n\right)} * f^{(-n)} \equiv 0 .
$$

Because of Lemma 1 , the function $p_{n}$ fulfills all the assumptions which were laid on $p$ in Section 1. We have therefore a clear notion of spectrum of an element in the class $F_{p_{n}}^{*}$, and the results in Section 1 , are true with $p$ substituted by $p_{n}$. A direct computation shows that if $f \in I(0)$ we have

$$
\widehat{f^{(-n)}}(t) \equiv \frac{1}{(i t)^{n}} \hat{f}(t)
$$

for $t \neq 0$, and for $t=0$ if the left hand member is defined by continuity. By use of (18) it follows that the class of functions $f^{(-n)}$, considered as elements of $F_{p_{n}}$, is not included in any of the regular maximal ideals in the class which correspond to $t \neq 0$, and by (16) it is not even included in the regular maximal ideal, which corresponds to $t=0$. Hence (17) has the consequence that the spectrum of $\varphi^{\left(m_{n}+n\right)}$, where this function now is considered as an element of $F_{p_{n}}^{*}$, is empty. By $B$ in Section 1 this implies that

$$
\varphi^{\left(m_{n}+n\right)} \equiv 0,
$$

almost everywhere. Hence $\varphi$ is equivalent to a polynomial of at most the degree $m_{n}+n-1$. This proves the proposition and hence also the theorem.

\section{Proof of Theorem 2.}

Lemma 4. If the additional assumptions 2 are fulfilled, then there exists a positive entire function 


$$
P(x)=\sum_{0}^{\infty} a_{2 n} x^{2 n}
$$

with every $a_{2 n} \geqq 0$, and such that for every $\varepsilon>0$

$$
[p(x)]^{2} \leqq x^{-2} P(x) \leqq P(x) \leqq[p(x(1+\varepsilon))]^{2}
$$

if $|x|$ is sufficiently large.

Proof. It is sufficient to prove that

$$
[p(x)]^{2} \leqq x^{-2} P(x) \leqq P(x) \leqq x^{5}[p(x)]^{2}
$$

if $x$ is sufficiently large. Firstly, the restriction to positive $x$ is a consequence of the fact that the functions are even. Secondly, for any $\varepsilon>0$ we have by Lagrange's mean value theorem and the convexity assumption in the additional assumptions 2

$$
\begin{aligned}
& \log p(x(1+\varepsilon))-\log p(x)=\log p\left(e^{\log x+\log (1+\varepsilon)}\right)-\log p\left(e^{\log x}\right) \\
& \geqq \log (1+\varepsilon) \cdot \frac{d}{d(\log x)} \log p(x)=\log (1+\varepsilon) \cdot \log x \cdot \frac{\frac{d}{d x} \log p(x)}{x^{-1} \log x} .
\end{aligned}
$$

Hence, using the last condition in the additional assumptions 2 we have for sufficiently large $x$

Starting from

$$
\frac{p(x(1+\varepsilon))}{p(x)} \geqq x^{\frac{5}{2}}
$$

$$
y=l_{0}(t) \equiv \lim _{t \rightarrow-\infty} 2 \log p\left(e^{t}\right)
$$

we form a sequence of tangents, with increasing slopes,

$$
y=l_{2 n}(t), \quad n=0,1, \ldots,
$$

to the convex function

$$
y=2 \log p\left(e^{t}\right),
$$

such that if $\left(t_{2 n+1}, y_{2 n+1}\right)$ denotes the point of intersection between

we have

$$
y=l_{2 n}(t) \quad \text { and } \quad y=l_{2 n+2}(t),
$$

$$
y_{2 n+1}=2 \log p\left(e^{t_{2 n+1}}\right)-1 .
$$

It can be proved, using the convexity, that

$$
l_{2 n}(t) \leqq l_{2 n+2}(t)-1, \quad \text { if } \quad t \geqq t_{2 n+3},
$$


and that

$$
l_{2 n}(t)-1 \geqq l_{2 n+2}, \quad \text { if } \quad t \leqq t_{2 n-1} .
$$

Using this it is easy to prove that

$$
\left[p\left(e^{t}\right)\right]^{2} \leqq e \sum_{n=0}^{\infty} e^{l_{2 n}(t)} \leqq \frac{e(3 e-1)}{e-1}\left[p\left(e^{t}\right)\right]^{2} .
$$

Then, let us consider

$$
l_{2 n}(t)=b_{2 n} t+c_{2 n},
$$

where obviously $b_{2 n} \geqq 0$. Let $m_{2 n}$ denote the smallest even integer which is $\geqq b_{2 n}$. We obtain from (20)

if $t>0$. Hence

$$
\left[p\left(e^{t}\right)\right]^{2} \leqq \sum_{n=0}^{\infty} e e^{m_{2 n} t+c_{2 n}} \leqq \begin{gathered}
e(3 e-1) \\
e-1
\end{gathered}\left[p\left(e^{t}\right)\right]^{2} e^{2 t}
$$

$$
[p(x)]^{2} \leqq \sum_{n=0}^{\infty} e^{c_{2 n}+1} x^{m_{2 n}} \leqq \frac{e(3 e-1)}{e-1}[p(x)]^{2} x^{2}
$$

if $x>1$. We therefore conclude that the function

fulfills (19).

$$
P(x)=1+\sum_{n=0}^{\infty} e^{c_{2 n+1}} x^{m_{2 n+2}}
$$

Proof of Theorem 2. We form the class $F_{P}^{2}$ of all measurable $f$, such that

$$
\int_{-\infty}^{\infty}|f(x)|^{2} P(x) d x<\infty .
$$

Lemma 4 and Cauchy-Schwarz' inequality give

$$
\left(\int_{-\infty}^{\infty}|f(x)| p(x) d x\right)^{2} \leqq \text { const. } \int_{-\infty}^{\infty}|f(x)|^{2} P(x) d x,
$$

which shows that $F_{P}^{2}$ is a subclass of $F_{p}$ equipped with a stronger topology. Using the Parseval relation we have for functions in $F_{P}^{2}$

$$
\int_{-\infty}^{\infty}|f(x)|^{2} P(x) d x=\frac{1}{2 \pi} \sum_{0}^{\infty} a_{2 n} \int_{-\infty}^{\infty}\left|\hat{f}^{(n)}(t)\right|^{2} d t,
$$

and this relation shows that the class $F_{P}^{2}$ might as well be characterized as the class of Fourier transforms in the $L^{2}$ sense of the class of functions $\hat{f}$ for which the right hand member of $(21)$ exists and is finite.

This shows that every $f \in F_{P}^{2}$, such that the corresponding $\hat{f}$ vanishes at $t=0$ together with all its derivatives, can be represented as a sum 


$$
f=f^{+}+f^{-},
$$

where $f^{+}$and $f^{-}$belong to $F_{P}^{2}$ and have Fourier transforms which vanish on $t \leqq 0$ and $t \geqq 0$, respectively. Obviously

$$
\lim _{\varepsilon \rightarrow 0}\left(f^{+} e^{i \varepsilon x}+f^{-} e^{-i \varepsilon x}\right)=f
$$

in the strong limit sense, and hence the closure of the class of $f \in F_{P}^{2}$ such that $\hat{f}$ vanishes identically in a neighborhood of $t=0$ contains every function $f \in F_{P}^{2}$ such that $\hat{f}$ vanishes together with all its derivatives at $t=0$.

The closure was then taken in the $F_{P}^{2}$ sense, but since the $F_{p}$ topology is weaker we have the result: The $F_{p}$ closure of any class of functions which contains every $f \in F_{p}$ such that $\hat{f}$ vanishes in a neighborhood of $t=0$, contains the whole class $F_{P}^{2} \cap I_{\infty}(0)$.

But since any closed primary ideal of type $I(0)$ can be characterized by relations

$$
\varphi * f \equiv 0,
$$

where $\varphi \in F_{p}^{*}$ and $\Lambda_{\varphi}$ consists only of the point $t=0$, the property $D$, $\S 1$, implies that $I(0)$ contains every function $f$, such that $\hat{f}$ vanishes on a neighborhood of $t=0$. Hence we have: $I(0)$ contains the whole class $F_{P}^{2} \cap I_{\infty}(0)$.

It remains only to show that $F_{P}^{2} \cap I_{\infty}(0)$ is dense in $I_{\infty}(0)$. To that end we shall prove:

a) Every $f \in I_{\infty}(0)$, such that $|f| p$ is bounded, can be approximated arbitrarily closely by elements in $F_{P}^{2} \cap I_{\infty}(0)$.

b) Every $f \in I_{\infty}(0)$ can be approximated arbitrarily closely by elements $g \in I_{\infty}(0)$ such that $|g| p$ is bounded.

Proof of a). Since $p$ is monotone, $f(x(1+\varepsilon))$ belongs to $I_{\infty}(0)$ for every $\varepsilon>0$, and $f$ can be approximated arbitrarily closely by these elements. But (19) gives

$$
\begin{aligned}
\int_{-\infty}^{\infty}|f(x(1+\varepsilon))|^{2} P(x) d x & \leqq \text { const. } \int_{-\infty}^{\infty}|f(x(1+\varepsilon))|^{2}[p(x(1+\varepsilon))]^{2} d x \\
& \leqq \text { const. } \sup |f| p \cdot \int_{-\infty}^{\infty}|f| p d x,
\end{aligned}
$$

and hence the functions $f(x(1+\varepsilon))$ belong to $L_{P}^{2} \cap I_{\infty}(0)$.

Proof OF b). It is easy to see that it is possible to approximate any $f \in I_{\infty}(0)$ arbitrarily closely by functions of the form

$$
g=f * h,
$$


where $h$ belongs to $F_{p}$, and has the property that $|h| p$ is bounded. By use of (2) it can be proved that then also $|g| p$ is bounded, and $g$ obviously belongs to $I_{\infty}(0)$.

\section{Some corollaries.}

TheOREM 4. If $p$ fulfills the additional assumptions 1 and if the function $f$ in $F_{p}$ has the property that

and

$$
\hat{f}(t)=0, \quad \text { if } \quad t \neq t_{0},
$$

$$
\hat{f}^{(n)}\left(t_{0}\right) \neq 0 \text {, }
$$

then all solutions in $F_{p}^{*}$ of the integral equation

$$
\varphi * f \equiv 0
$$

are polynomials of at most the degree $n-1$, multiplied by $e^{i t_{0} x}$.

If $p$ fulfills the additional assumptions 2 and if the function $f$ in $F_{p}$ has the property that $\hat{f}$ vanishes at $t=t_{0}$ together with all its derivatives, then

$$
\varphi * f \equiv 0
$$

for every $\varphi \in F_{p}^{*}$ such that $\varphi e^{-i t_{0} x}$ is an entire function of exponential type 0.

Proof. The first part follows at once from Theorem 1 using the fact that the primary closed ideal of functions $f_{0}$ in $F_{p}$ such that

is not included in $I_{n}\left(t_{0}\right)$.

$$
\varphi * f_{0} \equiv 0
$$

The second part is a consequence of Theorem 2 which shows that $I_{\infty}\left(t_{0}\right)$ has no proper primary closed sub-ideal. For that reason, if $\varphi$ has the form announced in the theorem, the ideal of all $f_{0}$ such that

$$
\varphi * f_{0} \equiv 0
$$

includes $I_{\infty}\left(t_{0}\right)$, and hence also $f$.

Starting from a function $p$ which satisfies the conditions in Section 1 we form the subspace $F_{p}^{0} \subset F_{p}^{*}$ which consists of all continuous functions which satisfy $\varphi(x)=o(p(-x))$ at infinity, and for which $\Lambda_{\varphi}$ consists at most of the point $t=0$. This subspace is closed, which is very easily seen, using the definition of spectrum and $C$, in Section 1.

The space of bounded linear functionals on $F_{p}^{0}$ can be identified with a certain class of bounded Borel measures on $-\infty<x<\infty$, and since $\mathrm{C}$ in Section 1 shows that only the values of the Fourier-Stieltjes transforms 


$$
\int_{-\infty}^{\infty} e^{-i t x} d \mu(x)
$$

in the neighborhood of $t=0$ are of any relevance as for the values of the corresponding functionals, we can as well assume that the measures are absolutely continuous. It turns out that the space of bounded linear functionals on $F_{p}^{0}$ is isomorphic with a space of equivalence classes of $F_{p}$ such that the values of the functional, corresponding to a certain equivalence class is given by

$$
\int_{-\infty}^{\infty} \varphi(-x) f(x) d x
$$

where $f$ is any element in the equivalence class.

By means of Theorem 4 we shall now prove two properties of the space $F_{p}^{0}$.

Theorem 5. If $p$ fulfills the additional assumptions 1 and if $\varphi$ is a function in $F_{p}^{0}$ which is not a polynomial, then the closure of the space of all linear combinations of translations of $\varphi$ contains all polynomials.

If $p$ fulfills the additional assumptions 2 , then the subspace of all polynomials is dense in $F_{p}^{0}$.

Proof. As for the first part, let $f$ be any function in $F_{p}$ (that is any bounded linear functional on $F_{p}^{0}$ ) such that

$$
\int_{-\infty}^{\infty} \varphi(t-x) f(x) d x \equiv 0 .
$$

Then $f$ has to be included in $I_{\infty}(0)$ by Theorem 4, first part. Hence

$$
\int_{-\infty}^{\infty} P(-x) f(x) d x=0
$$

for every polynomial $P(x)$, and thus the closure of the space of linear combinations of translations of $\varphi$ contains every polynomial.

If the second part were not true, it would be possible to find a not identically vanishing function $f \in F_{p}$ such that

$$
\int_{-\infty}^{\infty} P(-x) f(x) d x=0
$$

for every polynomial $P(x)$, whereas 


$$
\int_{-\infty}^{\infty} \varphi(-x) f(x) d x \neq 0
$$

for some $\varphi \in F_{p}^{0}$. But (22) implies that $f \in I_{\infty}(0)$, and therefore (23) and Theorem 4, second part, would be contradictory.

The second part of Theorem 5 gives apparently a solution of the wellknown Bernstein approximation problem (see for instance Mergelyan [4]) in certain cases, where the weight function is of non-quasianalytic type, i.e. satisfies (3).

\section{BIBLIOGRAPHY}

1. A. Beurling, Sur les intégrales de Fourier absolument convergentes et leur application à une transformation fonctionelle, Neuvième congrès des mathématiciens scandinaves tenu à Helsingfors 1938, Helsingfors, 1939, 345-366.

2. R. P. Boas, Entire functions, New York, 1954.

3. Y. Domar, Harmonic analysis based on certain commutative Banach algebras, Acta Math. 96 (1956), 1-66.

4. S. N. Mergelyan, Weighted approximation by polynomials, A. M. S. Translations (2) 10 (1958), 59-106.

5. R. Paley and N. Wiener, Fourier transforms in the complex domain, New York, 1934.

6. J. Wermer, On a class of normed rings, Ark. Mat. 2 (1954), 537-551. 